\title{
Rehabilitation Hand Exercise System with Video Games
}

\author{
Nurul Atikah Mazlan, Murizah Kassim, Saiful Izwan Suliman, Norsuzila Ya'acob \\ Faculty of Electrical Engineering, Universiti Teknologi MARA, 40450 UiTM Shah Alam, Malaysia \\ murizah@uitm.edu.my
}

\begin{abstract}
Rehabilitation hand exercises for stroke patients are continuing develop which assist with new technology. Some hospital still used manual coaches while exercising. Recent technology did discover assisted these activities with games and video are good for stroke patients to recover from the stroke. This paper presents a designed of game assists, motivates and helps stroke patients with hand movement problems. The game was interactively design with controllers and sensors for stroke patient rehabilitation process. A system consists of a controller that monitors the movement of hand by stroke patients is developed that interactively shows when patients handling games. It is embedded with heartbeat, hall and ultrasonic sensor to measure heartbeat rate, speed of hand movement and distances of hand pull from an object. A data analytics platform on SAS Viya is designed which presents real time data on heartbeat, speed, distance and movement of hands of the patient's exercised. Data is collected by sensors and the results able to analyze the progress of stroke patients which able to view online on cloud platform. This research is significant for patients and medical officers in monitoring stroke patients on hand problem for their rehabilitation progress.
\end{abstract}

\section{Key words: SAS Viya, Unity 3D, Hand Exercise Game}

\section{INTRODUCTION}

Usage of the video game in strokes patient rehabilitation process is not familiar yet but some of clinic or rehabilitation center had implemented it. The stroke rehabilitation process is one of the important stages for stroke's patient in order to recover the stroke. Without rehabilitation process, patients are unable to retrieve back their ability such as speaking, walking, dressing and others in a normal way [1,2]. Some patients are insisting to undergo a rehabilitation process due to several factors. The demotivating factor might come from the process that is too bored until they are not interested to finish the entire session. The duration of the normal rehabilitation process is one of the causes that make patients feel demotivated because it can take several years to fully recovered. Stroke can attack anyone. Not only an elderly people, but children also have the risk of stroke problem[3].

Not all of the activities suit the patient's mentality. Three problem statements were identified that lead to the development of this Hand Exercise with Video Games (HEVG). First, the traditional way of the rehabilitation process can demotivate them to undergo the therapy. Next, the existing method required a therapist that conducts the rehabilitation therapy to record the data manually without the help of sensors that can monitor patient heart rate, speed, and distance of hand movement. Third, the difficulties that faced by a therapist to analyze data in an organized way are one of the problems that should be solved in order to make sure the patient succeed in their therapy[4].

This research presents a designed rehab hand exercise system for hemiplegic stroke patients that suffered an upper limb disability. People that suffered these kinds of stroke had a greater loss in upper limb more than lower limb[5]. The patients in rehabilitation might use this HEVG game to help their recovery process. It can motivate the patient to move their hand and they can also enjoy playing the video game. It is an attractive way to attract patient interest to further their recovery process. The second priority users are children that suffer a stroke. This is because playing a video game is closer to children 's mentality more than elder people.Data analytics with interactive hand exercise video games will get the rate of the patient's hand movement. The game should record the data of the rate of hand movement and heartbeat rate. The HEVG game system is powered by Arduino UNO that process the sensor that attaches on it. The smart PC then submit the result to SAS Viya to do data analytics and displayed it on the graph.

\section{LITERATURE REVIEW}

Reviews on precious research related to the designed system are presented.

\subsection{Hand Exercise System}

Stroke can cause disability if patients are not well undergoing for the rehabilitation therapy. Stroke patients experienced physical changes in motor and sensory function which faced problem to do normal activities. Some of them faced loss ability to control the postural that lead to lack of safety while walking and contribute to the instability of body movement. Rehabilitation therapy is a must for stroke's patient to regain back their ability and motor skills[6]. The movement of limbs is important for stroke patient to gain back their usual ability from lose them forever.

Virtual reality (VR) is commonly used in rehabilitation therapy also for education that helps the development of motor skills for the patient if the used it repetitively or any learning process. Regular training for chronic post-stroke patients has been done by using virtual reality in rehabilitation therapy. The patients need to use two devices called CyberGlove and Rutgers Master II-ND (RMII) to control the simple game. The duration of training is about one and half hours for every virtual exercise session. The VR 
game is designed based on daily activities in the form of subtest that related to the improvement of motor skills such as pick up the objects. The result shows positive feedback as most patient that going through the test had an improvement in moving their fingers and thumb[7]. Figure 1 shows robotic glove to improve the finger movement that has been designed to study predefined recovery exercises system for after stroke hand rehabilitation. The robotic glove called intelligent haptic robotic glove (IHRG) acted as control architecture for dexterous grasping and manipulation. IHRG is used to support stroke human hand and for recovery activities. Exercises are divided into seven types that required the patient to move their fingers. It is a beneficial where stroke patients are able to do the therapies exercise without the help of a specialist which it is easily handled with glove and used it[8].

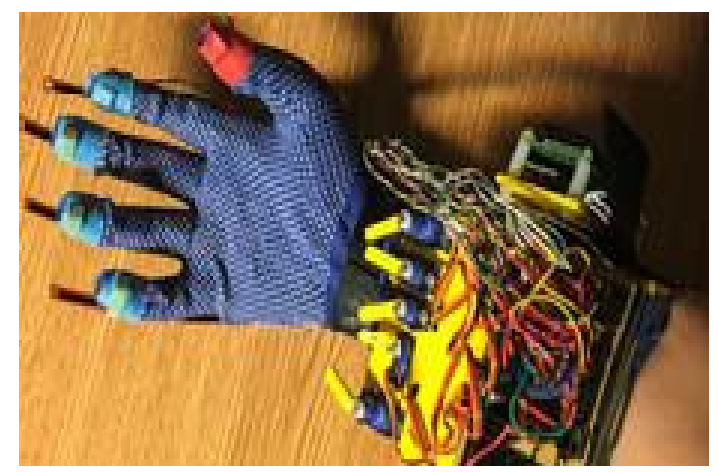

Figure 1: Intelligent Haptic Robotic Glove (IHRG)

\subsection{Interactive Video Game on Exercise}

Playing video game as a medium of exercise is the latest invent games in healthcare. A reseach method has presented a proposed a designed controller with exercise bicycle to measure stroke patients activities assisted by video games[9]. Another study shows a group of researcher from University of Ulster has developed a 3D Virtual Reality rehabilitation system. They are using marker-based augmented reality system where the marker is attached to real objects which enable the system to track the position and orientation of each object as it is moved. Many AR systems have been used that captured image of the real environment with computergenerated graphics to present a variety of game or task-driven scenarios to the user either in education of health $[10,11]$. A music Glove is introduced for a patient that suffered upper limb disability because of the stroke. The function of Music Glove is for the patients to practice the hand movement such as gripping movement by touching the sensor on the tip of the thumb to one of the other five sensors in time with music through a video game that displays scrolling notes on a screen. This method increased the motivation of a patient where they can perform hundreds of functional gripping movement in 30 minutes training session. This shows a good improvement in hand grasping[12]. Patients are motivated and enjoyed with the approach of Virtual Reality in exercise while playing the game. A device called Myo armband is tested on a person without health problem but has been introduced to strokes patient as it can be one of the therapy methods. The armband is worn by the users as when they move their hand, it is dynamically with the designed game. The game is designed by using Unity $3 \mathrm{D}$ software that is suitable for those with hand injury[13].

A Mobile Game-Assisted Rehabilitation System (GEAR) has been proposed as an exercise game to cater stroke problem. GEAR system consisted of a smart wearable wristband with sensor unit where it functions to detect the movement of patient's hand while playing a game. The user will wear the wristband for shoulder exercise and it interacted with a game installed on the smartphone. The game called 'pluck a fruit' and if the patient succeeds to pluck the fruit, it shows that they are able to replicate the instructed move well[14]. Another research presents a combination of an exercise game and an assistive exoskeleton is used as a tool of rehabilitation for children with Cerebral Palsy (CP). The knee extension exercise aim is to enable a more upright posture while from this exercise patients will repeat their knee movement. Game developed in this study called 'Cube Destroyer' where the user needs to pilot the spacecraft through intervals of ring and cubes. The results show positive feedback in knee extension where the participants successfully navigate through the game under exoskeleton assisted conditions[15].

\subsection{Rehabilitations Interactive Game}

A lot of interactive game had been invented recently for multipurpose such as engineering education and rehabilitation purpose[11]. One of the purpose of rehabilitation therapy has introduced low-cost Virtual Reality (VR) rehabilitation gaming system where a Microsoft Kinect ${ }^{\mathrm{TM}}$ camera is used to detect the upper limb movement. The focus of this VR game is to help stroke's patient that suffered upper limb disability to help them in hand movement. It will improve patient motor skills because this system promotes upper limb movement by using this VR system The patient can experience a virtual game such as reaching an object in the virtual system[16]. An invention called as Dance Mat was introduced in order to help the people that undergo the rehabilitation therapy. Dance Mat is a stepping game where the user needs to step on the mat to conduct the game. The game called "Chase a Groundhog" and "Catch a Butterfly". The "Chase a Groundhog" game, the groundhog will randomly appear in a hole and user needs to step on the mat based on the holes it appears. This will enhance the movement of lower limb such as leg so that this system is invented for someone with Cerebral Palsy to train their motor skills[17]. The game uses a Kinect device for motion capture which includes pre-build human body skeleton model that can be fitted accurately to the user's body length is introduced by the past researcher. The Kinect device adopts the RGB and 3D depth sensing camera for interacting user to a game environment. The game has presented body movement that controls the game and dynamic adaption module uses the patient's profile according to his progress to configure the game setting automatically. The system allows the patient to practice movements related to specific motor skills and interact with a virtual object in real time by multiple modalities[18].

The study was conducted on stroke's patient in one-year post-stroke with a plateau in motor recovery after conventional rehabilitation, video capture system(IREX) 
virtual reality system is used to capture the participant's whole body movement. The patient is able to see their body movement on a screen where their body movement is used to control the game. The patient will enjoy a game such as stepping up or down and snowboarding in 30 minutes. The result of this study shows the improvement in their body balancing by playing the games[19]. The Circus Challenge was introduced as a rehabilitation video game where it is a task-oriented activity that presents a series of gaming scenarios. It is based on the player movement as directed by the games to scores points in that game. The focus of this game is to move the upper limb such as arm and shoulder. Several of a game included in this game to attract player interest to continue their therapy. There are levels of difficulty and various game types. The player can interact with the video game by using a wireless controller[20].

\subsection{Data Analytics on Hand Exercise Game}

Data analytics platform is designed in this research for an easier task to analyze the patient's progress[21]. All data is organized in graphical form through SAS Viya platform. Collected data from patients activities ran by HEVG game are heart rates which recorded the hand movement until patients finished the games. Data analytics is important for doctors to monitor the patient's progress during the therapy. A rehabilitation game is proposed where not only it can monitor the exercise but it also provides a complete report of patients for a therapist. The analytics platform also functions to notify the therapist on the patient's ability, their motor skills and the improvement obtained by time. This helps the therapist to improve patient's ability and adjusting the daily exercise for them. Data are kept on private cloud which is easy access by doctors and enable efficient retrieval. Previous research presents a game called GEAR that stored data from the users in cloud database for analysis purpose. GEAR analytics developed by following a user-centric design approach where it is a web-based dashboard to empower clinicians with patient-specific data. The advantages of GEAR analytics are to propose appropriate follow-up treatments for the patients and facilitate decision making for therapist[22].

\section{METHODOLOGY}

\subsection{System Flow Chart}

Figure 2 shows the flow on the designed hand exercise game. It is designed by using hardware and software to make a complete HEVG game. This HEVG game is about user's hand movement is used to control the game and at the same time they control the HEVG game, the screen (Smart PC) is displaying the video game. The hand movement acts as a controller to the video game. Once the user had moved their hand, all the sensors work based on their function. After the user had finished the game, the score is displayed to inform the user about their score based on the game instruction whether it succeeds or not. User's data such as heart rate and distance of hand traveled are stored in the memory as they finished the game. The SAS Viya platform is used to organized data in graphical form for the data analytics.

\subsection{Block Diagram of HEVG Games}

The software, hardware, and component used must be emphasized to make sure the project is successful in order to complete the project (HEVG game). Figure 3 shows the block diagram of the HEVG game. First, we do the analysis on a component that should be used based on how the system functions. Ultrasonic sensor and hall sensor are used to measure the distance of user's hand can travel and the speed of hand movement. We used the pulse sensor to detect their heartbeat rate for medical purpose. Arduino UNO is used as a controller to control these sensors. Arduino UNO is a microcontroller based on software and programming.

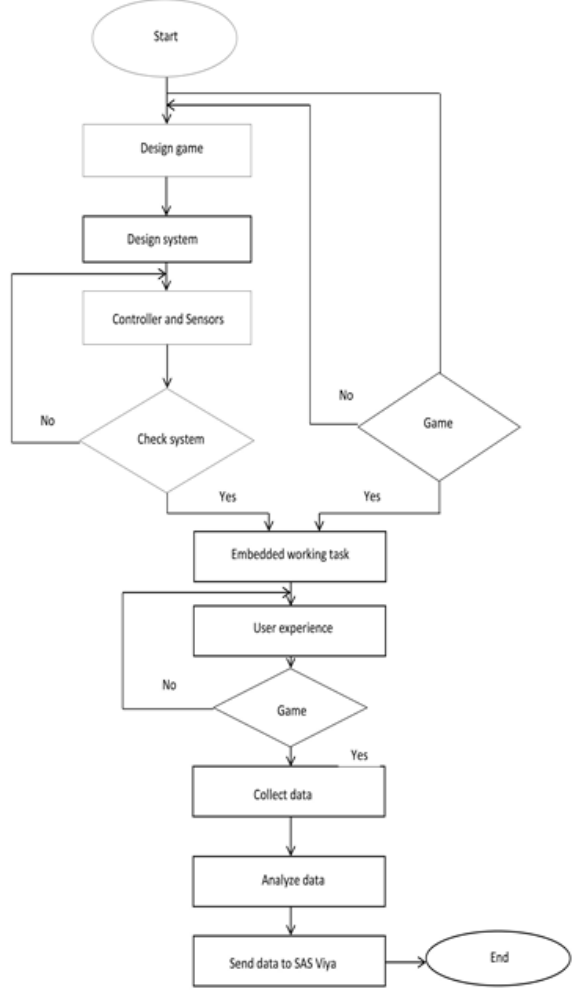

Figure 2: System Flow Chart

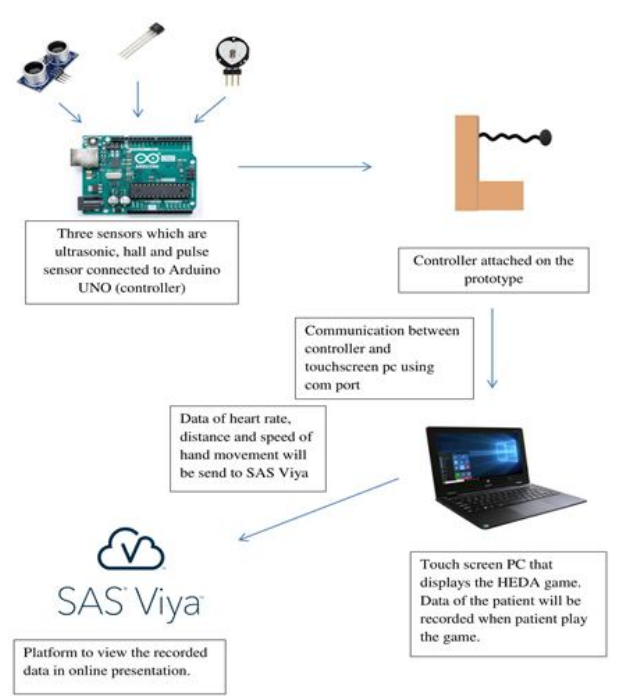

Figure 3: Block diagram of the system 
Arduino Uno is chosen as a controller that functions to read information from input devices used such as sensors. This controller is attached on the prototype to make sure all of the sensors embedded on the controller will work as user start to use the game. The sensor detects all the movement made by a user. Next, as user start to play the HEVG game, smart PC displays a video game that powered by Unity 3D game engines. The entire game interface is designed by using this software. The data is obtained from the patient's heartbeat rate, rate of hand movement and distance travelled by their hand after they finished the game. The data is stored in SAS Viya cloud storage for data analytical purpose. Finally, the therapist can analyze the patient data based on their interaction towards the game.

\subsection{Hardware}

The prototype in this project as shown in Figure 4 was made of wooden block where there is spring attached on it. The sensors were attached on this block. The ultrasonic sensor is attached at the top of the block and pulse sensor is attached at the end of the spring where there is holder that player will hold to control the game. The ultrasonic sensor is used to measure distance by using ultrasonic wave. Pulse sensor is used to determine the patient heartbeat rate. Hall sensor is used to detect the speed and the rate of hand movement. The accelerometer was used to determine the angle of player hand so that the video game move simultaneously as player starts to move their hand.

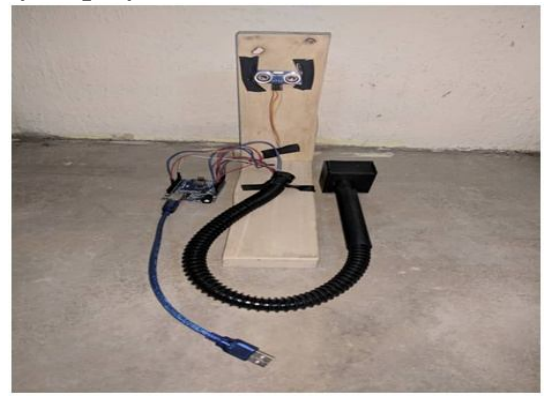

Figure 4: Hand Exercise Game device

\subsection{Software}

Three softwares are used in this project. First is Arduino software as shown in Figure 5 that is used to write a code for each sensor. The sensor used is ultrasonic, hall sensor, pulse sensor and accelerometer. All of the sensors were connected on Arduino board and the code was written on Arduino software. Next, after the code on Arduino is run, the output from accelerometer will control the video game. Accelerometer is working in three-axis such as when player move their hand the ball will move simultaneously. The game code is written on Unity 3D game engine as shown in Figure 6 that is open source engine that is free to use. The script or code is written by using $\mathrm{C \#}$ language. $\mathrm{C \#}$ language is written by using Visual Studio 2017 platform where the code from Arduino can be implemented in C\# language. Figure 7 shows the SAS Viya analytics platform. The data from the game is stored in the cloud and is presented in SAS Viya for data analytics. The SAS Viya allows keeping track all the information that is recorded. These data can be observed later on to see the performance of the user.

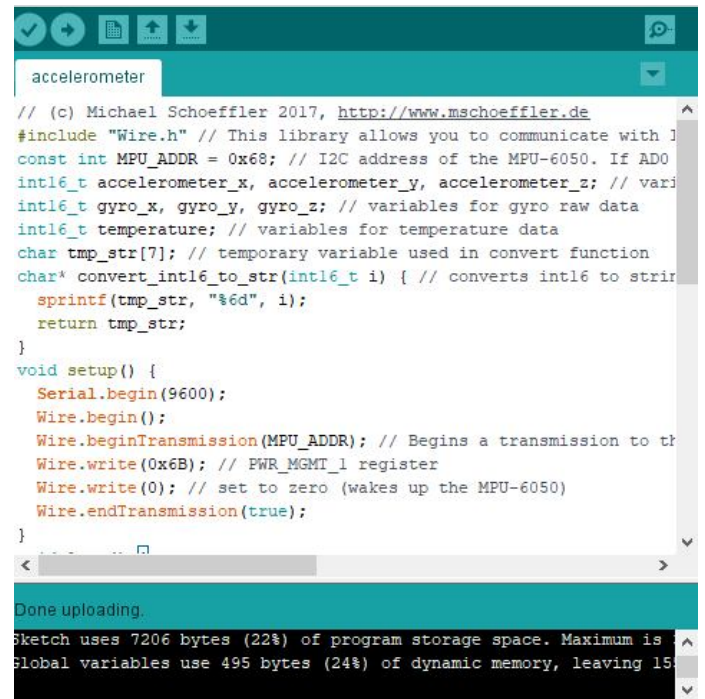

Figure 5: Example of Arduino software

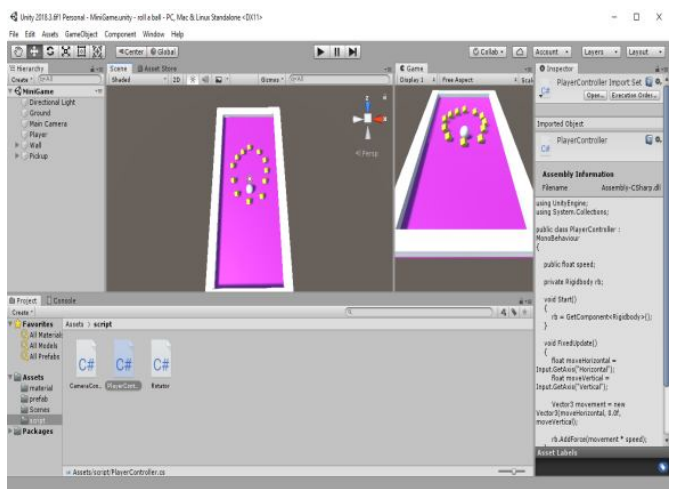

Figure 6: Unity 3D game engine

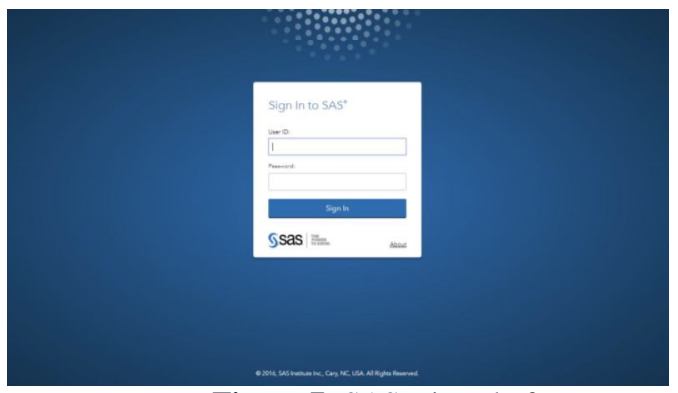

Figure 7: SAS Viya platform

\section{RESULTS AND DISCUSSIONS}

This part is the explanation of the project functionality such as prototype, video game and data analytics.

\subsection{Roll a Ball Video Game}

The game called Roll a Ball as shown in Figure 8 had been built by using Unity $3 \mathrm{D}$ game engine.

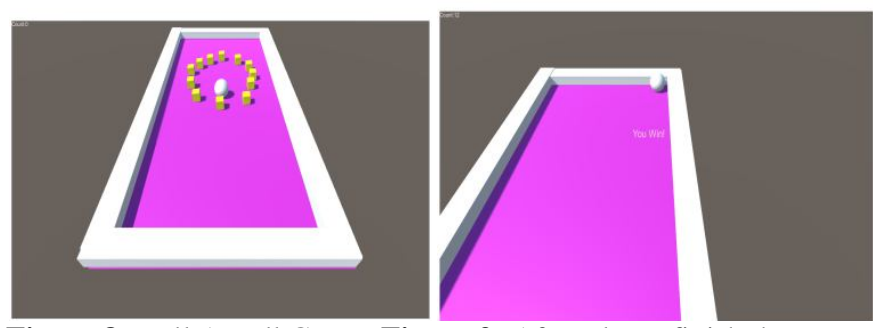

Figure 8: Roll A Ball Game Figure 9: After player finish the game 
This game required player to move their hand around to pick up the cube around the ball which act as player. The cube is arranged and rotates at the static position as where player is just simply move their hand towards the cube. The player will gain a score as their start to collect the cube. The player has to move their hand by moving the holder attached at the end of the spring for make the ball move. The game is easy as player just need to move their hand until the entire cube had been collected. The game will display text "You Win!" after player finish their game as in Figure 9.

\subsection{Circuit Design}

The circuit design involving the Arduino UNO and the sensors. The Arduino acted as the microcontroller. Arduino is used as it simplifies the circuit by just connecting the pin on the sensor to it. The sensors used including pulse sensor, hall sensor and ultrasonic sensor. It is needed to be embedded on the wooden block which is the project prototype. The connection is simple since the prototype just need to be attached on the PC and it is ready to play by user. The Arduino is placed beside the prototype as the breadboard connects the wire between Arduino and prototype. Figure 10 shows the connection of accelerometer on Arduino board as to test the component functionality and condition.

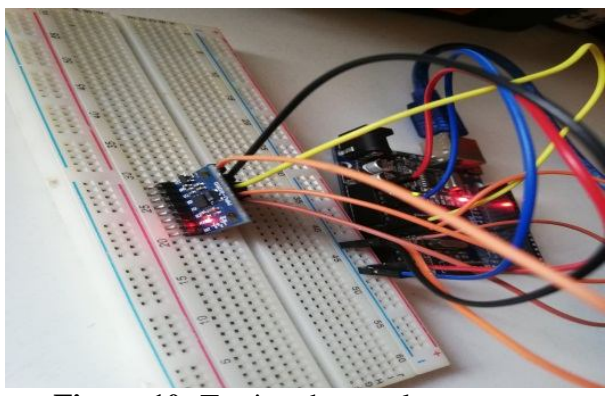

Figure 10: Testing the accelerometer

\subsection{Data Retrieval}

The output from the sensors is the input for controlling the video game. The data from the game such as score is stored in cloud for data analytics use. The sensors generate the output such as distance of hand travelled, the angle of hand movement and heart rate monitor by pulse sensor. The data from the game is stored in Microsoft Excel before it is export to the SAS Viya. The data is uploaded to SAS Viya to present the trend and patient progress based on how she can finish the game. After them playing the game, a set of data including score, player name, distance of hand travelled, speed and heart rate value is display in the form of graph. This will ease the therapist work to track their patient progress when they use normal rehabilitation and game type rehabilitation. SAS Viya is used as analytical platform because of faster processing for huge amounts of data and the most complex analytics, including machine learning, deep learning and artificial intelligence.

Table 1 shows the data of patient after they finished the game. A set of data including user's name, score, time, heartbeat rate and distance are recorded after user finish playing the game. Table 2 shows the summarized data of user after them playing the game. The data is summarized based on minimum, maximum, average and total for every element.
This data is used to display the bar chart graph to ease the therapist work to monitor their patient progress. Figure 11 presents the distance of hand exercise travelled by users on the frequency and the distance of player's hand sensed by ultrasonic sensor. Figure 12 shows the heartbeat rate data display while they playing the game.

Table 1: Video Game Data

\begin{tabular}{|c|c|c|c|c|}
\hline Name & Score & Time(s) & Heartbeat Rate(bpm) & Distance (m) \\
\hline User1 & 12 & 60 & 151 & 12 \\
\hline User2 & 12 & 75 & 100 & 11 \\
\hline User3 & 12 & 50 & 150 & 11 \\
\hline User4 & 12 & 55 & 115 & 13 \\
\hline User5 & 12 & 65 & 130 & 15 \\
\hline
\end{tabular}

Table 2:. Summarized Data on SAS Viya

\begin{tabular}{|c|c|c|c|c|}
\hline Name & Minimum & Maximum & Average & Sum \\
\hline Distance & 11 & 15 & 12.4 & 62 \\
\hline Heartbeat Rate & 100 & 151 & 129.2 & 646 \\
\hline Score & 12 & 12 & 12 & 60 \\
\hline Time & 50 & 75 & 61 & 305 \\
\hline
\end{tabular}

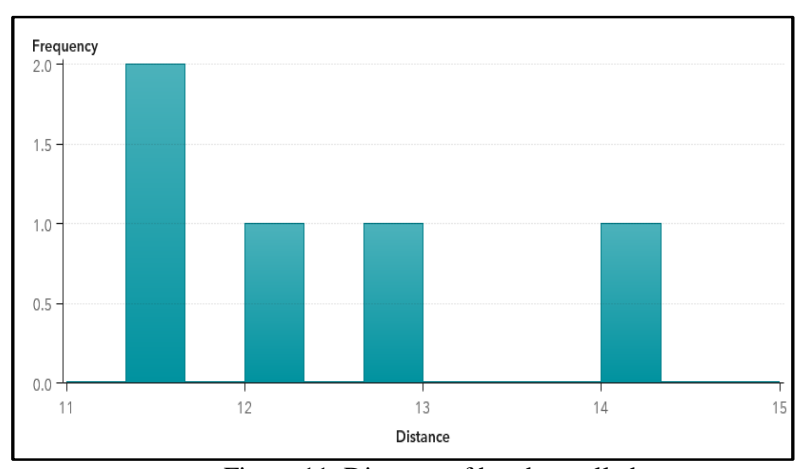

Figure 11. Distance of hand travelled

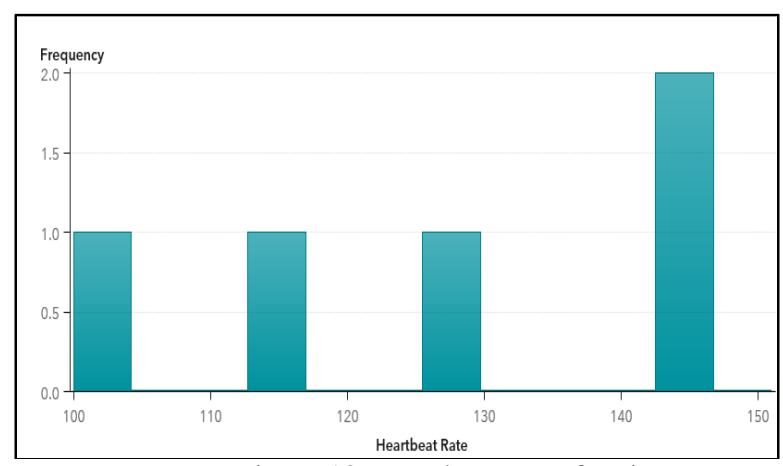

Figure 12. Heartbeat rate of patient

\section{CONCLUSION}

The rehabilitation process is the must for someone that suffered the stroke. The process helps them to regain and retrieve back their normal abilities. They must undergo the rehabilitation process in order to treat the problem. The HEVG game is one of the ways to help them undergo their therapy with joyful and safe way. It can encourage and motivate them to regain their abilities and lost skills by experiencing a new way of the rehabilitation process with the 
implementation of a video game in rehabilitation activities. The objectives of this project are successfully achieved. First, a game that can motivate and helps stroke patients with hand problems which interactively communicate with design controllers and sensors or stroke patient rehabilitation process is well designed. Second, a system consist of a controller that monitors the movement of hand by stroke patients embedded with heartbeat, hall and ultrasonic sensor to measure heartbeat rate, the speed of hand movement and distances of hand from an object had been designed. Finally, a data analytics platform on SAS Viya in presenting on heartbeat, speed, distance and movement of hands of the patient's data collected by sensors had been developed to analyze the progress of stroke patients and presented on online and cloud platform.

\section{ACKNOWLEDGMENT}

Authors would like to thanks to Research Management Institute, Universiti Teknologi MARA, UiTM Shah Alam for the support grant in publishing this paper.

\section{REFERENCES}

[1] M. A. Latif, H. M. Yusof, S. Sidek, M. Shikhraji, and M. Safie, "A gaming-based system for stroke patients physical rehabilitation," in 2014 IEEE Conference on Biomedical Engineering and Sciences (IECBES), 2014, pp. 690-695. https://doi.org/10.1109/IECBES.2014.7047594

[2] S. F. Tan, F. Ahmedy, and S. Y. Teh, "Designing and developing predictive rehabilitation management system for patient registry in northern borneo," International Journal of Advanced Trends in Computer Science and Engineering, vol. 8, pp. 3588-3595, 2019. https://doi.org/10.30534/ijatcse/2019/142862019

[3] H. J. Fullerton, Y. W. Wu, S. Sidney, and S. C. Johnston, "Risk of recurrent childhood arterial ischemic stroke in a population-based cohort: the importance of cerebrovascular imaging," Pediatrics, vol. 119, pp. 495-501, 2007.

[4] B. Jabber, P. Sai Venkat, K. Sri Sai Nikhil, and B. Lakshmi Avinash, "A novel sampling approach for balancing the data and providing health care management system by government," International Journal of Advanced Trends in Computer Science and Engineering, vol. 8, pp. 2753-2761, 2019.

https://doi.org/10.30534/ijatcse/2019/12862019

[5] B. H. Dobkin, "Strategies for stroke rehabilitation," The Lancet Neurology, vol. 3, pp. 528-536, 2004. https://doi.org/10.1016/S1474-4422(04)00851-8

[6] D. Novokhrestova, E. Kostyuchenko, and E. Kosenko, "Automation of speech quality assessment in speech rehabilitation," International Journal of Advanced Trends in Computer Science and Engineering, vol. 8, pp. 3026-3030, 2019.

https://doi.org/10.30534/ijatcse/2019/58862019

[7] S. V. Adamovich, A. S. Merians, R. Boian, J. A. Lewis, M. Tremaine, G. S. Burdea, M. Recce, and H. Poizner, "A virtual reality-based exercise system for hand rehabilitation post-stroke," Presence: Teleoperators \& Virtual Environments, vol. 14, pp. 161-174, 2005.

[8] N. Popescu, V. Ciobanu, A. Ghidel, F. Dinu, and D. Popescu, "Predefined recovery exercises system for after stroke hand rehabilitation," in 2016 International Conference and Exposition on Electrical and Power Engineering (EPE), 2016, pp. 331-336.
https://doi.org/10.1109/ICEPE.2016.7781357

[9] M. Kassim and M. N. H. M. Said, "Data analytics on interactive indoor cycling exercises with virtual reality video games," in Proceedings - 2018 4th International Conference on Control, Automation and Robotics, ICCAR 2018, 2018, pp. 321-326.

[10] J. W. Burke, M. McNeill, D. K. Charles, P. J. Morrow, J. H. Crosbie, and S. McDonough, "Augmented reality games for upper-limb stroke rehabilitation," in 2010 Second International Conference on Games and Virtual Worlds for Serious Applications, 2010, pp. 75-78.

https://doi.org/10.1109/VS-GAMES.2010.21

[11] M. Kassim and M. T. H. Md Zubir, "Design of augmented reality for engineering equipment in education," International Journal of Advanced Trends in Computer Science and Engineering, vol. 8, pp. 2773-2781, 2019. https://doi.org/10.30534/ijatcse/2019/15862019

[12] D. K. Zondervan, N. Friedman, E. Chang, X. Zhao, R. Augsburger, D. J. Reinkensmeyer, and S. C. Cramer, "Homebased hand rehabilitation after chronic stroke: Randomized, controlled single-blind trial comparing the MusicGlove with a conventional exercise program," Journal of Rehabilitation Research \& Development (JRRD) V53 (4), 2016. https://doi.org/10.1682/JRRD.2015.04.0057

[13] R. Lipovský and H. A. Ferreira, "Hand therapist: A rehabilitation approach based on wearable technology and video gaming," in 2015 IEEE 4th Portuguese Meeting on Bioengineering (ENBENG), 2015, pp. 1-2.

[14] M. Rahman, B. Wadhwa, A. Kankanhalli, Y. C. Hua, C. K. Kei, L. J. Hoon, S. Jayakkumar, and C. C. Lin, "GEAR analytics: A clinician dashboard for a mobile game assisted rehabilitation system," in 2016 4th International Conference on User Science and Engineering (i-USEr), 2016, pp. 193-198.

[15] T. C. Bulea, Z. F. Lerner, A. J. Gravunder, and D. L. Damiano, "Exergaming with a pediatric exoskeleton: facilitating rehabilitation and research in children with cerebral palsy," in 2017 International Conference on Rehabilitation Robotics (ICORR), 2017, pp. 1087-1093. https://doi.org/10.1109/ICORR.2017.8009394

[16] N. Norouzi-Gheidari, M. F. Levin, J. Fung, and P. Archambault, "Interactive virtual reality game-based rehabilitation for stroke patients," in 2013 International Conference on Virtual Rehabilitation (ICVR), 2013, pp. 220221.

[17] V. Kozyavkin, B. Kachmar, O. Terletskyy, O. Kachmar, and I. Ablikova, "Stepping games with Dance Mat for motor rehabilitation," in 2013 International Conference on Virtual Rehabilitation (ICVR), 2013, pp. 174-175. https://doi.org/10.1109/ICVR.2013.6662108

[18] S. Saini, D. R. A. Rambli, S. Sulaiman, M. N. Zakaria, and S. R. M. Shukri, "A low-cost game framework for a homebased stroke rehabilitation system," in 2012 International Conference on Computer \& Information Science (ICCIS), 2012, pp. 55-60. https://doi.org/10.1109/ICCISci.2012.6297212

[19] K. E. Laver, B. Lange, S. George, J. E. Deutsch, G. Saposnik, and M. Crotty, "Virtual reality for stroke rehabilitation," Cochrane database of systematic reviews, 2017.

[20] J. Serradilla, J. Shi, Y. Cheng, G. Morgan, C. Lambden, and J. A. Eyre, "Automatic assessment of upper limb function during play of the action video game, circus challenge: validity and sensitivity to change," in 2014 IEEE 3nd International Conference on Serious Games and Applications for Health (SeGAH), 2014, pp. 1-7. 
https://doi.org/10.1109/SeGAH.2014.7067073

[21] S. Handoyo, A. Widodo, W. H. Nugroho, and I. N. Purwanto, "The implementation of a hybrid fuzzy clustering on the public health facility data," International Journal of Advanced Trends in Computer Science and Engineering, vol. 8, pp. 3549-3554, 2019. https://doi.org/10.30534/ijatcse/2019/135862019

[22] M. Rahman, A. Kankanhalli, B. Wadhwa, Y. C. Hua, C. K. Kei, L. J. Hoon, S. Jayakkumar, and C. C. Lin, "GEAR: A mobile game-assisted rehabilitation system," in 2016 ieee international conference on healthcare informatics (ichi), 2016, pp. 511-516.

https://doi.org/10.1109/ICHI.2016.92 